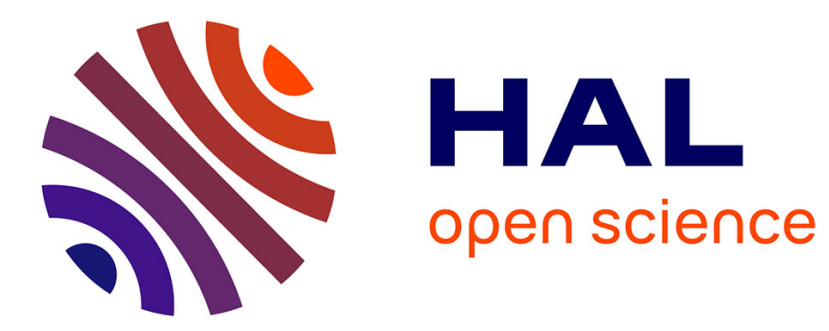

\title{
Phase diagram and critical temperatures of nearly half-filled quasi one-dimensional conductors
}

\author{
Chr. Seidel, V.N. Prigodin
}

\section{To cite this version:}

Chr. Seidel, V.N. Prigodin. Phase diagram and critical temperatures of nearly half-filled quasi one-dimensional conductors. Journal de Physique Lettres, 1983, 44 (10), pp.403-409. 10.1051/jphyslet:019830044010040300 . jpa-00232209

\section{HAL Id: jpa-00232209 https://hal.science/jpa-00232209}

Submitted on 1 Jan 1983

HAL is a multi-disciplinary open access archive for the deposit and dissemination of scientific research documents, whether they are published or not. The documents may come from teaching and research institutions in France or abroad, or from public or private research centers.
L'archive ouverte pluridisciplinaire HAL, est destinée au dépôt et à la diffusion de documents scientifiques de niveau recherche, publiés ou non, émanant des établissements d'enseignement et de recherche français ou étrangers, des laboratoires publics ou privés. 
Classification

Physics Abstracts

$71.30-74.40-74.10$

\title{
Phase diagram and critical temperatures of nearly half-filled quasi one-dimensional conductors
}

\author{
Chr. Seidel $\left(^{*}\right)$ and V. N. Prigodin
}

A. F. Ioffe Physico-Technical Institute, Academy of Sciences of the USSR, 194021 Leningrad, USSR

(Reçu le 3 avril 1982, révisé le 12 mars 1983, accepté le 25 mars 1983)

\begin{abstract}
Résumé. - Nous considérons l'influence du remplissage de bande et du couplage transversal sur les températures de transition (diélectrique et supraconductrice) de conducteurs quasi unidimensionnels. Ces influences sont similaires. Une transition métal-supraconducteur existe soit pour $|\delta \mu|>\delta \mu_{\mathrm{c}}$, soit pour $W_{\perp}>W_{\mathrm{c}}\left(\right.$ avec $\left.W_{\mathrm{c}}^{2} \simeq \varepsilon_{\mathrm{F}} T_{\mathrm{P}}\right)$ pourvu que $0<g_{4}<\left|g_{3}\right|$. A partir de cette théorie nous discutons la situation expérimentale des conducteurs organiques(TMTSF) ${ }_{2} \mathrm{X}$.

Abstract. - Considering a nearly half-filled quasi 1D conductor, variations in bandfilling and in transverse coupling are shown to have similar influence on the critical temperatures of the dielectric (CDW, AF) and superconductor (SS, TS) transitions. Provided $0<g_{4}<\left|g_{3}\right|$ a metal-to-superconductor transition occurs at $|\delta \mu|>\delta \mu_{\mathrm{c}}$ where $g_{4}\left(\delta \mu_{\mathrm{c}}\right)=0$, or at $W_{\perp}>W_{\mathrm{c}}$ where $W_{\mathrm{c}}^{2} \simeq \varepsilon_{\mathrm{F}} T_{\mathrm{P}}$, respectively. Using the theoretical results we discuss the experimental situation for the new organic conductors (TMTSF) ${ }_{2} \mathrm{X}$.
\end{abstract}

Recently there has been considerable interest in the investigation of some organic crystals of the type (TMTSF) ${ }_{2} \mathrm{X}$, where $\mathrm{X}$ is e.g. $\mathrm{PF}_{6}, \mathrm{AsF}_{6}, \mathrm{ClO}_{4}, \mathrm{ReO}_{4}, \mathrm{NO}_{3}$ [1]. At low temperatures the majority of these quasi one-dimensional (1D) conductors undergoes a metal-to-insulator (M-I) transition. Nevertheless in several compounds (e.g. $\mathrm{X}=\mathrm{PF}_{6}, \mathrm{AsF}_{6}$ ) there is no indication for a usual Peierls transition. Concerning (TMTSF) ${ }_{2} \mathrm{PF}_{6}$ it has been pointed out that the M-I transition has unusual magnetic character, i.e. the low temperature phase is expected to be an antiferromagnetic one [2]. On the other hand there are members of the (TMTSF) ${ }_{2} \mathrm{X}$ family where the M-I transition is also a structural one $\left(\mathrm{X}=\mathrm{ReO}_{4}, \mathrm{NO}_{3}\right)$. However it is, at least mainly, not an electronically driven Peierls transition but an order-disorder transition of the anions [3]. The most interesting feature shown by some representatives of this new class of organic conductors doubtless is the existence of bulk superconductivity. For instance (TMTSF) ${ }_{2} \mathrm{PF}_{6}$ becomes superconducting under pressure $(p \simeq 6 \mathrm{kbar})$ with a pressure-dependent critical temperature $T_{\mathrm{c}} \lesssim 1.3 \mathrm{~K}$ [4], and (TMTSF) ${ }_{2} \mathrm{ClO}_{4}$ shows a metal-to-superconductor (M-S) transition at ambient pressure $\left(T_{\mathrm{c}} \simeq 1.4 \mathrm{~K}\right)$ [5]. Due to the perfect $2: 1$ stoichiometry the compounds in question are commensurate having a 3/4 filled band. However as a result of the presence of a

$\left({ }^{*}\right)$ Permanent address : Institut für Polymerenchemie « Erich Correns », Akademie der Wissenschaften der DDR, 1530 Teltow-Seehof, GDR. 


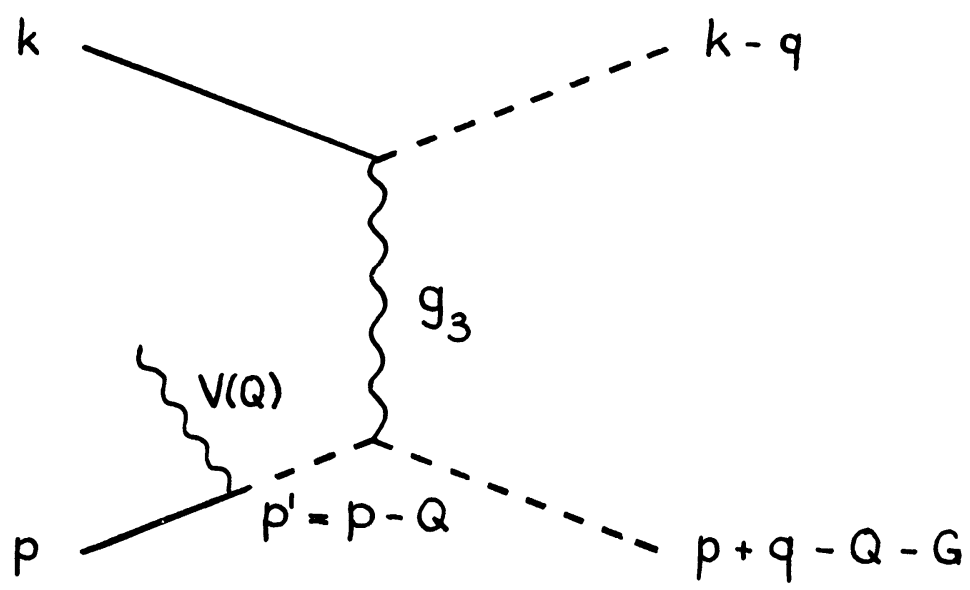

Fig. 1. - Modified U-process in the $3 / 4$ filled (TMTSF) ${ }_{2} \mathrm{X}$ compounds.

weak dimerization potential $V(Q), Q=(\pi / a, 0,0)$ the real situation is more complex [1]. In particular, umklapp $(\mathrm{U})$ processes become important when the potential $V(Q)$ is taken into account. An example of such U-processes is shown in figure 1. Owing to $V(Q)$ all the four electrons can be near the Fermi surface. But because of the high excited virtual intermediate state $\left(p^{\prime} \simeq-1 / 3 k_{\mathrm{F}}\right)$ whose Green function is

$$
G\left(p^{\prime}, \omega\right) \simeq\left(\omega+v_{\mathrm{F}} \frac{2}{3} k_{\mathrm{F}}\right)^{-1} \sim \frac{1}{\varepsilon_{\mathrm{F}}}
$$

the corresponding coupling constant $\tilde{g}_{3}$ is small. Assuming $V \sim \Delta_{\mathrm{D}} \ll \varepsilon_{\mathrm{F}}$, where $\Delta_{\mathrm{D}}$ is the dimerization gap (that is pressure dependent), in the order of magnitude we obtain

$$
\tilde{g}_{3}^{0} \simeq \frac{\Delta_{\mathrm{D}}}{\varepsilon_{\mathrm{F}}} g_{3}^{0}
$$

where $g_{3}^{0}$ is the coupling corresponding to U-processes in the half-filled band case.

In a recent paper we studied the influence of commensurability effects on the phase diagram of quasi 1D conductors with a nearly half-filled band [6]. Including the deviation of the Fermi energy from the middle of the conduction band as an additional characteristic energy a modified renormalization group $(R G)$ approach was suggested to study the effect of U-processes.

Although application of pressure should not have an important influence on bandfilling in one-chain materials like (TMTSF) ${ }_{2} \mathrm{X}$, in our opinion the fundamental results of the latter approach can be also used to describe the behaviour of such systems under pressure. In principle the bandfilling parameter $\delta \mu=v_{\mathrm{F}} \delta k_{\mathrm{F}}$ introduced in the theory is an accurate measure for the fulfilling of the condition $\mathbf{G} \simeq 2 \mathbf{q}_{0}$ where $\mathbf{G}$ is a reciprocal lattice vector, $\mathbf{q}_{0}$ the nesting vector of the Fermi surface. As discussed above, in the organic conductors under consideration there occurs a modified U-process $\hat{g}_{3}$ which gives an important contribution only if $\mathbf{G} \simeq 2 \mathbf{q}_{0}-Q$, i.e. $\delta \mu$ is measured with reference to the $3 / 4$ filled band. While in exactly 1D systems the relation between $\mathbf{G}$ and $\mathbf{q}_{0}$ is determined by bandfilling alone, in quasi 1D systems a dependence on interchain coupling appears. A small transversal coupling is enough to remove the perfect nesting of the Fermi surface except when the band is half-filled. The best nesting will occur for a wave vector $\overline{\mathbf{q}}_{0}$ where $\overline{\mathbf{q}}_{0}$ connects two inflection points of the Fermi surface and can be substantially different from $\mathbf{q}_{0}$ [7]. So, enlarging the corrugation of the Fermi surface the equality $\mathbf{G} \simeq 2 \overline{\mathbf{q}}_{0}-Q$ can be disturbed also for $\delta \mu=0$, and the U-processes $\tilde{g}_{3}$ will be suppressed. Providing $W_{\|} \gg W_{\perp}$ 
where $W_{\|}, W_{\perp}$ are the longitudinal and transversal bandwidths, respectively, the deviation of $\overline{\mathbf{q}}_{0}$ from $\mathbf{q}_{0}$ is expected to be linear to $W_{\perp}$. The corresponding coefficient depends on spectrum and bandfilling in detail. In this sense ignoring the complex nature of the pressure dependence one can think that the phase diagram of the commensurate quasi 1D conductors under consideration is similarly influenced by $\delta \mu$ (doping) and $\delta W_{\perp}$ (pressure).

Considering the $\delta \mu$-dependence let us summarize the main results of the theory. Taking into account backward scattering $\left(g_{1 \|}, g_{1 \perp}\right)$, forward scattering $\left(g_{2}\right)$ and U-processes $\left(g_{3}\right)$ the corresponding second-order RG equations are (the $g_{i}$ 's are measured in units $2 \pi v_{\mathrm{F}}, \hbar=k_{\mathrm{B}}=1$ )

$\xi \frac{\mathrm{d} g_{1 \|}}{\mathrm{d} \xi}=2 g_{1 \perp}^{2}+2 g_{1 \|} g_{1 \perp}^{2}$

$\xi \frac{\mathrm{d} g_{1 \perp}}{\mathrm{d} \xi}=2 g_{1 \|} g_{1 \perp}+g_{1 \|}^{2} g_{1 \perp}+g_{1 \perp}^{3}$

$\xi \frac{\mathrm{d} g_{2}}{\mathrm{~d} \zeta}=g_{1 \perp}^{2}+g_{1 \|} g_{1 \perp}^{2}-\left[1+\left(\frac{4 \beta}{\xi}\right)^{2}\right]^{-1}\left[g_{3}^{2}\left(g_{1 \|}-2 g_{2}\right)+g_{3}^{2}\right]$

$\xi \frac{\mathrm{d} g_{3}}{\mathrm{~d} \xi}=g_{3}\left(g_{1 \|}-2 g_{2}\right)+g_{3}\left(g_{1 \|}^{2}+g_{2}^{2}-g_{1 \|} g_{2}\right)+$

$$
\begin{aligned}
& +\left[1+\left(\frac{2 \beta}{\xi}\right)^{2}\right]^{-1}\left[3 g_{3}\left(g_{2}^{2}-g_{1 \|} g_{2}\right)+\frac{1}{2} g_{3}^{3}\right] \\
& +\left[1+\left(\frac{4 \beta}{\xi}\right)^{2}\right]^{-1}\left[g_{3}\left(g_{1 \|}-2 g_{2}\right)+\frac{1}{2} g_{3}^{3}\right],
\end{aligned}
$$

where

$$
\xi=T / E_{0}, \quad \beta=|\delta \mu| / E_{0}, \quad g_{i}\left(E_{0}\right)=g_{i}^{0}
$$

and $E_{0}$ is the cutoff parameter. The coupling constants determined by equation (2) exhibit the following properties. In the high temperature range $T>|\delta \mu|$ the half-filled band behaviour is maintained. However at $T \simeq|\delta \mu|$ a drastic change occurs in the behaviour of the system. Namely, (i) the coupling $g_{4}=g_{1 \|}-2 g_{2}$ becomes constant for $T<|\delta \mu|$ (equal to $\bar{g}=g_{4}(|\delta \mu|)$ ), and (ii) the coupling $g_{3}$ is irrelevant in the low temperature range. Thus one can think that at $T<|\delta \mu|$ the system shows the same behaviour as in the absence of U-processes. Their only effect consists in a renormalization of the coupling $g_{4}^{0}$ to $\bar{g}$. Starting from the behaviour of the coupling constants the dependence of the critical exponents $v$ of several generalized susceptibilities $\chi$ on bandfilling can be obtained. There is a characteristic energy $\Delta$ being in the order of the gap in the charge-density spectrum at half-filledness $\left(\Delta \sim\left|g_{3}\right|^{1 / 2} \mathrm{e}^{1 /\left|g_{3}\right|} W_{\|}\right)$below which $\bar{g}_{4}$ is in the vicinity of the fixed point -1 for $g_{4}^{0}<\left|g_{3}^{0}\right|$. For $\delta \mu$ up to $|\delta \mu| \simeq \Delta$ the known half-filled band behaviour is approximately maintained. Considering charge-density wave (CDW), antiferromagnetic (AF), singlet- (SS) and triplet-superconductor (TS) instabilities the divergence of $\chi_{\mathrm{CDW}, \mathrm{AF}}$ was shown to be reduced with increasing $|\delta \mu|>\Delta$ while the superconductor response functions are enhanced.

Taking into account a weak kinetic transverse coupling characterized by the corrugation $W_{\perp}\left(W_{\perp} \ll T_{0}\right)$, in the mean field approximation (MFA) the transition temperature is given by

$$
T_{\mathrm{c}}=T_{0}\left(\frac{W_{\perp}}{T_{0}}\right)^{-2 / v(\delta \mu)}
$$

where $T_{0}$ is a characteristic temperature below which $1 \mathrm{D}$ susceptibilities show power law behaviour. In the order of magnitude $T_{0}$ is equal to $\Delta$. The $v(\delta \mu)$ 's are the above discussed critical 

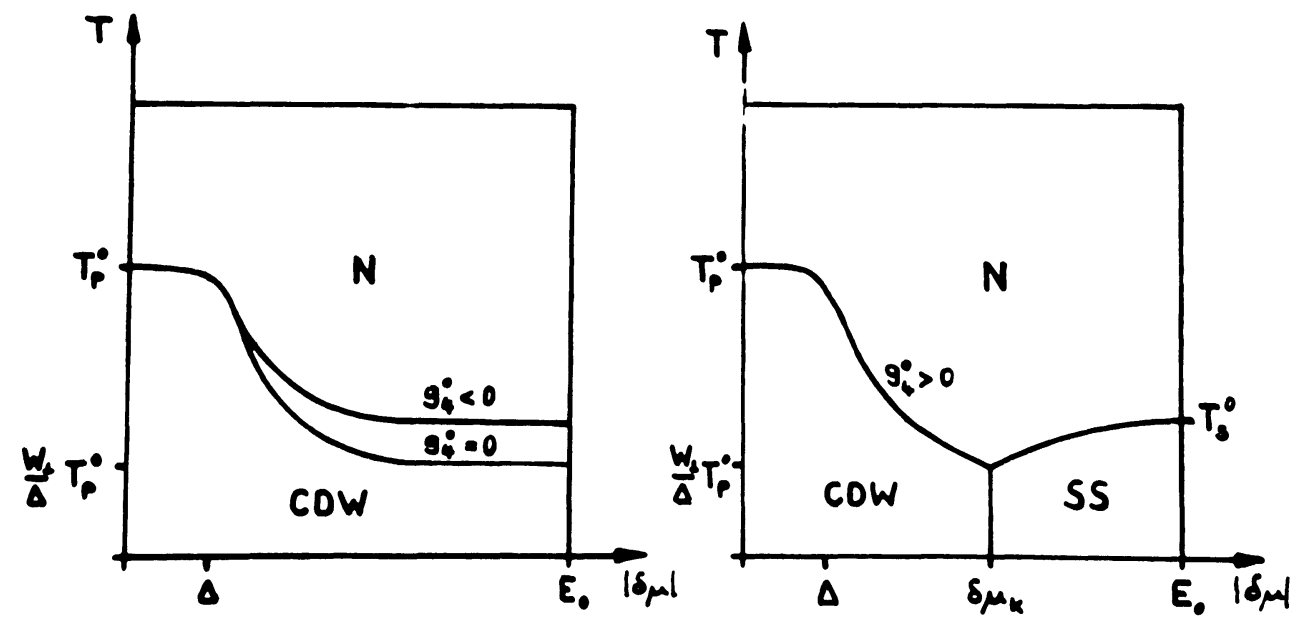

Fig. $2 a$. - Transition temperature dependence on bandfilling at $W_{\perp} \ll T_{0}\left(g_{1 \|}^{0}<\left|g_{1 \perp}^{0}\right|\right)$.

exponents of the 1D response functions. In agreement with the MFA the phase showing the most divergent susceptibility is realized in the system. Using the corresponding expressions for the $v(\delta \mu)$ 's [6] finally the result can be represented as phase diagram in the $T-\delta \mu$ plane. The case $g_{1 \downarrow}^{0}<\left|g_{1 \perp}^{0}\right|$ is shown in figure $2 a$. $\mathrm{N}$ denotes normal (metallic) phase. Equation (3) yields $T_{\mathrm{p}}^{0} \simeq W_{\perp}$. For $g_{4}^{0}>0$ there is a critical deviation $\delta \mu_{\mathrm{c}}$. At $|\delta \mu|>\delta \mu_{\mathrm{c}}$ the SS susceptibility becomes divergent with a higher degree than the CDW one, and the superconductor phase will be realized in the system.

At $g_{1 \|}^{0} \geqslant\left|g_{1 \perp}^{0}\right|$ a similar phase diagram is obtained. In this case AF and TS instabilities compete. (We note that for $g_{1 \|}=g_{1 \perp}=g_{1}>0$ the CDW (SS) instability becomes divergent

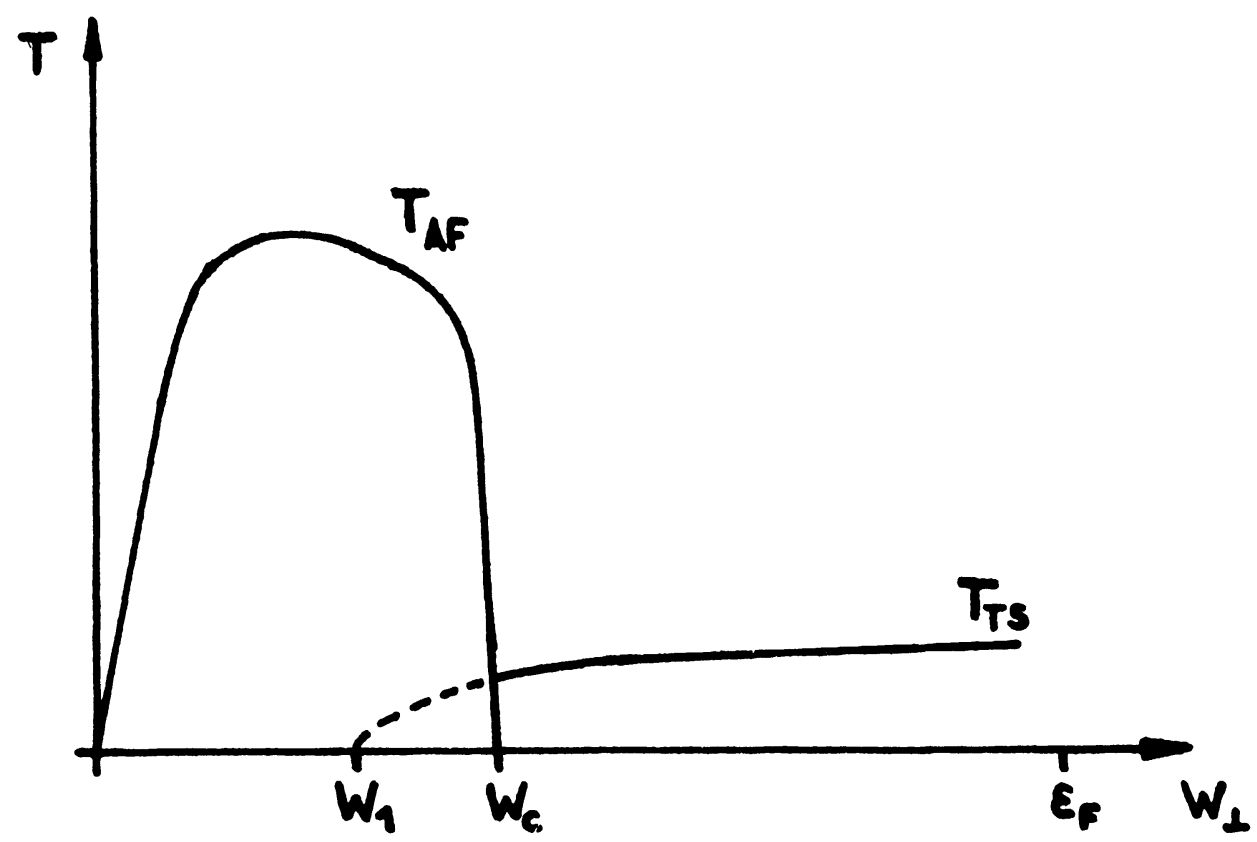

Fig. 2b. - Transition temperature dependence on transverse bandwidth at $W_{\perp} \gtrsim T_{0}\left(g_{1}^{0}>0\right)$. 
with the same degree as the AF (TS) one). Since the critical exponents appearing here are smaller by one compared to the case $g_{1 \|}^{0}<\left|g_{1 \perp}^{0}\right|$ the transition temperatures $T_{\mathrm{c}}(\delta \mu)$ are considerably lowered. At $\delta \mu=0$ we have $T_{\mathrm{AF}}^{0}=T_{n}^{0} W_{1} / T_{0}$. Due to the spin dependence of coupling $g_{1}$ the spin-density contribution $v_{6}$ occurring in the corresponding exponents $v$ at $g_{1 \|}^{0}>\left|g_{1 \perp}^{0}\right|$ is not identical to one, as provided in reference [6]. Consequently, in table I of reference [6] we have to replace -1 by $-2+v_{6}^{ \pm 1}$ where the signs + and - correspond to CDW, SS and AF, TS instabilities, respectively. In general we have [8]

$$
v_{6}=\left(\frac{1+g_{1 \|}^{*}}{1-g_{1 \|}^{*}}\right)^{1 / 2}
$$

where the saturation value $g_{1 \|}^{*}$ at $T \rightarrow 0$ according to equation (2) is given by

$$
g_{1 \|}^{*}=\left[\left(g_{1 \|}^{0}\right)^{2}-\left(g_{1 \perp}^{0}\right)^{2}\right]^{1 / 2}, \quad g_{1 \|}^{0}>\left|g_{1 \perp}^{0}\right| \text {. }
$$

So the critical temperatures $T_{\mathrm{AF}}, T_{\mathrm{TS}}$ maintain a nonzero value $T_{\mathrm{c}} \simeq T_{\mathrm{AF}}^{0}\left(W_{\perp} / T_{0}\right)^{2 / g_{1 \|}^{*}-2}$ at $|\delta \mu|=\delta \mu_{\mathrm{c}}$. The degeneration between TS/SS and AF/CDW instabilities disappears also as a result of finite transverse coupling. Then the constant $g_{1 \|}=g_{1}$ does not vanish at $T \rightarrow 0$, even though the remaining value is small. Thus TS and AF phases are enhanced whereas SS and CDW ones are suppressed. Thereby one can see that $T_{\mathrm{c}}\left(\delta \mu_{\mathrm{c}}\right)>0$.

Despite many difficulties to give a more detailed description of the complex nature of the interaction in real quasi 1D solids it is useful to discuss the relations between the coupling constants introduced above and several known interaction processes. Taking into account (i) the short range $\left(g_{\mathrm{C}}>0\right)$ and long range $(U>0)$ parts of the Coulomb interaction, (ii) the short range interaction originated by the exchange of phonons $\left(g_{\phi}<0\right)$ we have

$$
\begin{aligned}
& g_{1}^{0}=g_{\phi}+g_{\mathrm{C}} \\
& g_{2}^{0}=g_{\phi}+U \\
& g_{3}^{0}=g_{\phi}+g_{\mathrm{C}} \\
& g_{4}^{0}=-g_{\phi}+g_{\mathrm{C}}-2 U
\end{aligned}
$$

where

$$
g_{\phi}=-\frac{2 \lambda^{2}}{\omega\left(2 k_{\mathrm{F}}\right)} \frac{N_{0}}{4}, \quad N_{0} \simeq \frac{2}{\pi v_{\mathrm{F}} a^{2}}
$$

We note that in $g_{\phi}$ only interactions with longitudinal phonon modes (along the chain or stack direction, i.e. including also intermolecular phonons in the latter case) are taken into account. Due to the quasi one-dimensionality of the system under consideration the interchain interaction corresponding to the exchange of transversal phonons is assumed to be weak. In principle such processes can be considered as a contribution to the transverse coupling. We remember that according to equation (1) the coupling $g_{3}^{0}$ corresponding to half-filled band U-processes is reduced to $\tilde{g}_{3}^{0}$ in (TMTSF) ${ }_{2} \mathrm{X}$ as a result of the smallness of the dimerization gap $\Delta_{\mathrm{D}}$.

In the case of (TMTSF) ${ }_{2} \mathrm{PF}_{6}$ there are no indications on strong CDW fluctuations. Previous studies on the possible ground states of 1D conductors [8] suggest that such a phase can only occur when the spin dependence of $g_{1}$ is important. Formally this fact can be described by introducing a constant $g_{M}$ originated by an exchange of magnetic excitations. The AF phase exists only at $g_{1}^{0}>0$, i.e. when the Coulomb repulsion is strong and the electron-phonon interaction weak. As pointed out above, the system undergoes a M-S transition at $|\delta \mu|>\delta \mu_{\mathrm{c}}$ only if $g_{4}^{0}>0$. From equations (5) one can see that the simultaneous realization of $g_{4}^{0}>0$ and $g_{1}^{0}>0$ 
requires either a strong screening of the long range Coulomb interaction or a strong magnetic interaction.

As mentioned above the MFA expression (3) is only correct if $W_{\perp} \ll T_{0}$ where $T_{0}$ is in the order of $T_{\mathrm{c}}^{0}\left(T_{\mathrm{AF}}^{0} \simeq 12 \mathrm{~K}\right)$. However, recent experimental results indicate a relative strong transverse coupling at least in (TMTSF) ${ }_{2} \mathrm{PF}_{6}$ [9], and the MFA should cautiously be used for the compounds under consideration. Considering the RG equations for a quasi $1 \mathrm{D}$ system, i.e. when an additional characteristic energy $W_{\perp}$ occurs, Prigodin and Firsov [10] studied the dependence of the transition temperatures on $W_{\perp}$ in the interesting range $W_{\perp} \gtrsim T_{0}$. Following this approach and taking into account U-processes, their influence on several fluctuations was investigated by Kimura [11] we get the following expressions (in the further study we suppose that U-processes are not suppressed at $W_{\perp} \neq 0$ )

$$
\begin{aligned}
& T_{\mathrm{SS}}=T_{1} \exp \left[\frac{2}{3 g_{1}(\delta)-g_{4}(\delta)}\right], \\
& T_{\mathrm{TS}}=T_{1} \exp \left[\frac{2}{-g_{1}(\delta)-g_{4}(\delta)}\right], \\
& T_{\mathrm{P}}=T_{1} \exp \left[\frac{2}{3 g_{1}(\delta)+g_{4}(\delta)-2\left|g_{3}(\delta)\right|}\right], \\
& T_{\mathrm{AF}}=T_{1} \exp \left[\frac{2}{-g_{1}(\delta)+g_{4}(\delta)-2\left|g_{3}(\delta)\right|}\right],
\end{aligned}
$$

where

$$
\begin{gathered}
T_{1}\left(W_{\perp}\right) \sim W_{\perp}\left(W_{\perp} / \varepsilon_{\mathrm{F}}\right)^{v}, \quad v=0\left(g_{2}^{0^{2}}\right) \ll 1 \\
\delta=\ln \frac{T_{1}}{\varepsilon_{\mathrm{F}}} \simeq \ln \frac{W_{\perp}}{\varepsilon_{\mathrm{F}}} .
\end{gathered}
$$

Thereby one has to bear in mind that the dielectric transitions are suppressed at $W_{\perp}>W_{\mathrm{c}}$ where

$$
W_{\mathrm{c}}^{2} \simeq \varepsilon_{\mathrm{F}} T_{\mathrm{P}, \mathrm{AF}}^{0},
$$

because an increasing corrugation of the Fermi surface removes the electron-hole symmetry (due to terms in the order of $W_{\perp}^{2} / \varepsilon_{\mathrm{F}}^{2}$ )

$$
\varepsilon(k)-\mu=-\left[\varepsilon\left(k-q_{0}\right)-\mu\right] .
$$

For (TMTSF) ${ }_{2} \mathrm{PF}_{6}$ equation (9) yields $W_{\mathrm{c}} \simeq 7 \times 10^{2} \mathrm{~K}$. The transverse bandwidth at ambient pressure was estimated to be $W_{\perp}^{0} \simeq 2 \times 10^{2} \mathrm{~K}$ [12], i.e. under pressure $p \approx 6 \mathrm{kbar} W_{\perp}$ should be enlarged by a factor 3 .

Analysing equation (7) one can see that the behaviour of $T_{c}$ is not essentially changed in comparison with the results discussed in the first part of the paper. Now as before the AF phase exists at $g_{1}^{0}>0$, the CDW one at $g_{1}^{0}<0$, and a M-S transition can occur if $0<g_{4}^{0}<\left|g_{3}^{0}\right|$. For a repulsive interaction the behaviour of the transition temperatures is schematically shown in figure $2 b$. The parameter $W_{1}$ is determined by $g_{1}\left(\delta_{1}\right)=-g_{4}\left(\delta_{1}\right)$. (In Fig. $2 b$ we suppose $W_{1}<W_{\mathrm{c}}$ ). Compared to the phase diagram shown in figure $2 a$ the dielectric transition is more sharply suppressed here.

Finally we note : (i) The U-process $\tilde{g}_{3}$ will be also suppressed when the dimerization gap is reduced. However the pressure dependence of $\Delta_{\mathrm{D}}$ is not known. (ii) The experimentally obtained lowering of the M-S transition temperature with increasing pressure [12] cannot be explained by 
the mechanisms investigated above. It is possible that impurity effects are important here. This problem will be studied in a forthcoming paper. (iii) The M-S transition should be also driven by doping, perhaps the critical temperature will not be suppressed as under pressure.

After this paper was completed we learned about a work by V. J. Emery et al. [13] who pointed out that in particular the dimerization gap $\Delta_{\mathrm{D}}$ is a measure of the relevance of U-processes and should be correlated with the critical pressure for superconductivity.

\section{Acknowledgments.}

We would like to thank Prof. Yu. A. Firsov for his interest in the study. We are also indebted to Prof. K. Carneiro for an informative discussion on the physics of the new organic conductors.

\section{References}

[1] Bechgaard, K., Jacobsen, C. S., Mortensen, K., Pedersen, H. J. and Thorup, N., Solid State Commun. 33 (1980) 1119.

[2] Mortensen, K., Tomkiewicz, Y., Schultz, T. D. and Engler, E. M., Phys. Rev. Lett. 46 (1981) 1234.

[3] Pouget, J. P., Moret, R., Comés, R. and Bechgaard, K., J. Physique Lett. 42 (1981) L-543.

[4] Jérome, D., Mazaud, A., Ribault, M. and Bechgaard, K., J. Physique Lett. 41 (1980) L-95.

[5] Bechgaard, K., Carneiro, K., Olsen, M., Rasmussen, F. B. and Jacobsen, C. S., Phys. Rev. Lett. 46 (1981) 852.

[6] Seidel, Chr. and Prigodin, V. N., J. Low Temp. Phys. 48 (1982) 85.

[7] Jafarey, S., Phys. Rev. B 16 (1977) 2584.

[8] Prigodin, V. N. and Firsov, Yu. A., Zh. Eksp. Teor. Fiz. 71 (1976) 2252 (Engl. transl. Sov. Phys. JETP 44 (1976) 1187).

[9] Jacobsen, C. S., Tanner, D. B. and BechgaArd, K., Phys. Rev. Lett. 46 (1981) 1142.

[10] Prigodin, V. N. and Firsov, Yu. A., Zh. Eksp. Teor. Fiz. 76 (1979) 1602 (Engl. transl. Sov. Phys. JETP 49 (1979) 813).

[11] Kimura, M., Progr. Theor. Phys. 53 (1975) 955.

[12] Parkin, S. S. P., Ribault, M., Jérome, D. and Bechgaard, K., J. Phys. C 14 (1981) 5305.

[13] Emery, V. J., Bruinsma, R. and Barisic, S., Phys. Rev. Lett. 48 (1982) 1039. 